\title{
Tratamiento interdisciplinario de paciente adulto con trastornos de la articulación temporomandibular. Reporte de caso clínico.
}

\section{Interdisciplinary treatment of an adult patient with temporomandibular joint disorders. Clinical case report.}

\author{
Ramos-Montiel Ronald ${ }^{1}$, Brito-Zúñiga Cristian ${ }^{1}$, Puebla-Ramos Lorenzo ${ }^{2}$, Pinos-Soria Gabriela ${ }^{3}$ \\ ${ }^{1}$ Coordinador del posgrado de Ortodoncia de la Universidad Católica de Cuenca. \\ ORCID ID: https://orcid.org/0000-0002-8066-5365 \\ ${ }^{1}$ Residente del posgrado de Ortodoncia de la Universidad Católica de Cuenca. \\ ORCID ID:https://orcid.org/0000-0003-1814-7572 \\ ${ }^{2}$ Especialista en Ortodoncia. Hospital infantil de México Federico Gómez. Ciudad de México. \\ ORCID ID:https:orcid.org/0000-0002-0527-9990 \\ ${ }^{3}$ Especialista en Rehabilitación Oral, Universidad Andrés Bello, Santiago de Chile. \\ ORCID ID: https://orcid.org/0000-0002-2539-7230 \\ *rramosm@ucacue.edu.ec
}

\begin{abstract}
Resumen
Las alteraciones de la articulación temporomandibular son una de las principales causas por las que los pacientes acuden a la consulta odontológica con el afán de mitigar sus síntomas y signos. Aunque es complejo divisar las causas de esta afección, con una secuencia ordenada de examinación podemos acertar al diagnóstico. El tratamiento interdisciplinario de ortodoncia fija para corregir la posición dental con el propósito de coordinar las arcadas y rehabilitación oral para reponer la estructura dental perdida es una opción viable para lograr una estabilidad oclusal que a su vez acompañe a una posición ortopédica y sin alteraciones de la articulación temporomandibular.
\end{abstract}

Palabras clave: Desórdenes temporomandibulares, Articulación temporomandibular, Ortodoncia.

Temporomandibular disorders are some of the main reasons why patients go to the dentist seeking to relieve their signs and symptoms. Even though, it is hard to perceive the causes of this pathology, with a correct sequence of the examination protocol we will be able to diagnose the problem. Interdisciplinary treatment between orthodontics to improve dental position, aiming arch coordination and oral rehabilitation to build up the loss of tooth structure is a suitable option to reach oclusal stability which turns into an orthopedic position of the temporomandibular joint.

Key words: Temporomandibular disorders, Temporomandibular joint, Orthodontics.

\section{INTRODUCCIÓN}

Las disfunciones temporomandibulares (DTM) son un subconjunto dentro de la condición del dolor orofacial, caracterizado por el dolor que emana, en diferentes intensidades, de los músculos de la masticación, de la articulación, del oído, la cabeza, entre otros. En este tiempo moderno entre el 50 y $60 \%$ de la población presenta algún grado de disfunción temporomandibular o molestias relacionadas con trastornos del sistema estomatognático. ${ }^{1}$ Los factores emocionales de estrés, ansisedad, depresión, fatiga, insomnio y dolores crónicos son los que pueden desencadenar hábitos parafuncionales y tensión muscular que predisponen a la aparición de trastornos temporomandibulares. ${ }^{2}$

El bruxismo es uno de los factores más destacados asociados al estrés y ansiedad, es un acto inconsciente y parafuncional en el que el paciente aprieta y rechina los dientes, produciendo así un desgaste patológico de los mismos. $^{3}$

Por otro lado, la alteración en la oclusión es considerada un factor etiológico para los DTM, pero su relación es compleja y no está completamente definida. La pérdida dental y los desórdenes oclusales no están directamente 
asociados como factores causales de los desórdenes temporomandibulares. ${ }^{4}$ Además, las interferencias oclusales tampoco van a generar siempre DTM, gracias a la capacidad de la articulación temporomandibular de adaptarse a ellas, pero en pacientes con historial de DTM las interferencias desencadenan una recurrencia mucho mayor y más aguda de los síntomas.

Los modelos etiopatogénicos de los desórdenes temporomandibulares no tiene un uso práctico para los pacientes dado a su multifactoriedad. También su etiología idiopática impone un enfoque personalizado del paciente para el diagnóstico, planificación y tratamiento de las alteraciones temporomandibulares. Los principales signos que se presentan en los DTM son, limitaciones en la apertura, ruidos articulares y dolor de los músculos masticatorios o de la articulación, como principal síntoma por el que los pacientes buscan tratamiento para cesar esta patología. ${ }^{5}$

El RDC (Research Diagnosis Criteria) es el sistema estandarizado de examinación más ampliamente usado tanto para individuos asintomáticos y para pacientes con DTM. Este método incluye una examinación clínica con Axis I y un test psiquiátrico con Axis II, ambos métodos han demostrado ser instrumentos diagnósticos válidos para los DTMs. ${ }^{6}$ Además de los métodos clínicos, la resonancia magnética y la tomografía computarizada son considerados el gold standard para detectar desordenes intraarticulares de la ATM. Los métodos de diagnóstico manuales son una colaboración entre ortodoncistas y fisioterapistas para realizar exámenes de detección de alteraciones de la ATM antes de los tratamientos de ortodoncia.

Diferentes tratamientos para los DTMs son aplicados a los pacientes, desde tratamientos iniciales como una correcta comunicación con el paciente sobre su diagnóstico, que crea un efecto placebo, la motivación por el buen pronóstico del tratamiento, cambios en la textura de la dieta, instrucciones de cómo cambiar su actividad oral mediante la auto observación y la auto corrección de hábitos y parafunciones, además, terapias físicas y manuales mediante ultrasonido, TENS, laser, kinesioterapia, masajes localizados, prescipción de fármacos como antirreumáticos no esteroides, analgésicos o relajantes musculares y también el uso de splints oclusales ya sean de relajación, distracción o de reposición son diferentes terapias que pueden ser utilizadas, por lo general en combinación, para la corrección de alteraciones a nivel de la ATM. ${ }^{7}$

Pero el tratamiento ortodóncico puede ser visto desde dos puntos de vista, en el que el tratamiento de ortodoncia tiene un impacto negativo en el desarrollo de signos y síntoma de DTMs y por otro lado que la ortodoncia está relacionada como tratamiento para los DTMs. El uso de aparatos fijos de ortodoncia parece ser eficaz para el tratamiento del dolor articular y muscular, pero no para los ruidos articulares. ${ }^{8}$ En los pacientes que inician el tratamiento de ortodoncia con alteraciones de la articulación temopromandibulare por ejemplo con desplazamiento anterior del disco, se puede lograr una posición mejor del disco interarticular. ${ }^{9}$ También es sabida hace muchos años atrás el efecto de remodelado óseo de la cavidad glenoidea y del cóndilo que tiene como efecto el tratamiento de ortodoncia, de manera que, si los arcos quedan bien coordinados, con un correcto engranaje, adecuadas llaves de desoclusión, el estado alterado de la ATM al inicio del tratamiento puede verse mejorado siempre y cuando logremos una estabilidad de la oclusión que acompañará y pacificará a la articulación temporomandibular. ${ }^{10}$

Constantemente encontramos signos clínicos de trastornos de la articulación temporomandibular, sin embargo, el paciente muy pocas veces sabe que las presenta, el paciente por lo general acude a consulta por problemas que son más evidentes a la vista, principalmente piezas desalineadas, desgastadas, la mala apariencia, problemas estéticos, etc, razón por la que tenemos que estar preparados para reconocer cuál fue el origen de esa alteración, diagnosticarla y estructurar un plan de tratamiento.

\section{REPORTE DE CASO}

Paciente masculino de 30 años de edad, acude a la consulta porque le disgusta el aspecto desgastado de sus dientes, presenta dificultad en la masticación y manifiesta molestias eventuales al abrir su boca.

El paciente refiere que en su trabajo está sometido constantemente a tensiones, padece dolores matutinos frecuentes de la articulación temporomandibular que se irradian de la zona maseterina hacia la región temporal. A la exploración física responde con dolor tipo gatillo al momento de palpar la zona alrededor de la ATM y se puede divisar una luxación con reducción del cóndilo tanto en apertura como en cierre y una disminución de la apertura bucal. A la evaluación extraoral se observa un perfil recto y biotipo mesofacial. (Figura 1) En el análisis clínico intraoral observamos que el paciente conserva todas sus piezas dentales y que la pérdida de estructura dental es por hábitos parafuncionales, tanto de morderse las uñas, objetos y bruxismo, presenta una mordida borde a borde, relación canina I bilateral y tejidos blandos gruesos, en la arcada superior presenta una completa giroversión de la pieza 2.5 y en la arcada inferior presenta un apiñamiento moderado. (Figura 2)

El estudio cefalométrico reveló que es un paciente clase III esqueletal por un prognatismo, con un crecimiento 


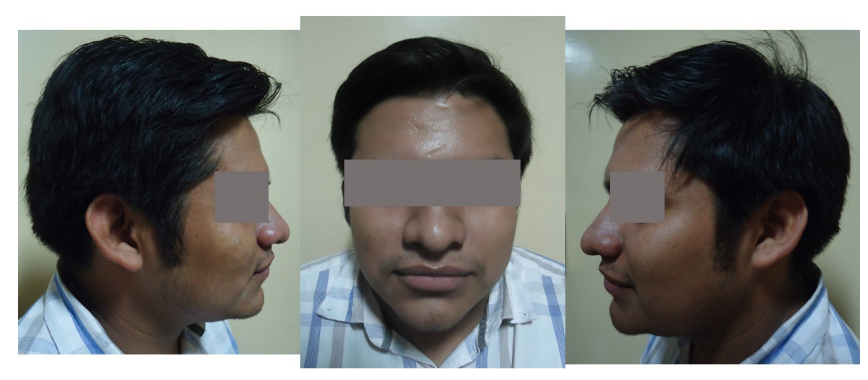

Fig. 1.

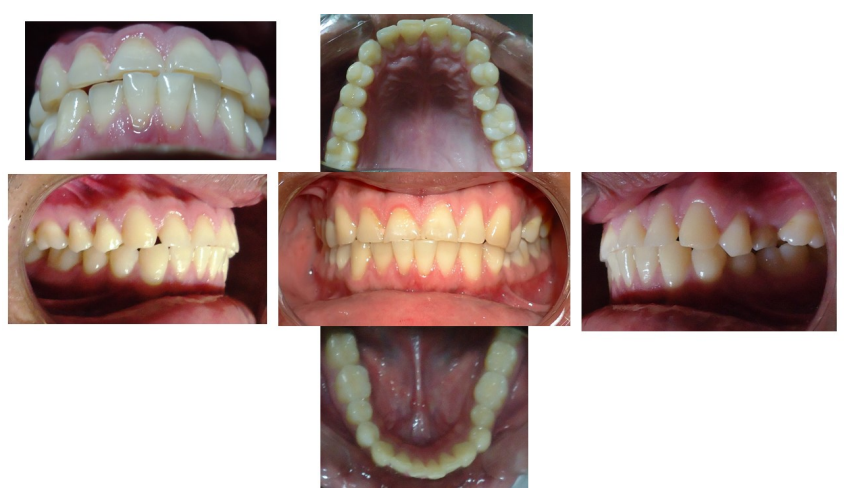

Fig. 2 .

normodivergente y biproinclinación dentoalveolar de los incisivos.

El plan de tratamiento que se eligió fue alinear y nivelar, luego la extracción de los segundos premolares de los cuatro cuadrantes para conservar el perfil del paciente y la utilización de biomecánica con fuerzas diferenciadas para lograr una buena relación interincisiva y conservar la clase I canina bilateral. Todo el proceso se lo realizó con levantes de mordida de resina en los primeros molares superiores para facilitar la biomecánica, para la reprogramación y pacificación de la ATM. El cierre de los espacios se los realizó con la combinación de cadenetas elásticas y resortes DKL. (Figura 3) Al finalizar el tratamiento de ortodoncia se realizó la reconstrucción de los bordes incisales con resina para mejorar las guías de desoclusión. (Figura 4) Al paciente se le colocó una retención fija superior e inferior y adicional se le envió con una guarda protectora acrílica superior para que lo utilice en las noches y apacigüe la articulación temporomandibular ya que en las noches es cuando se produce la descarga de las tensiones acumuladas durante el día. (Figura 5)

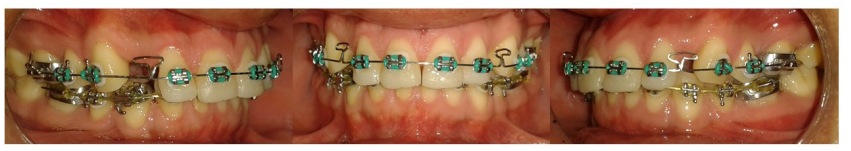

Fig. 3 .

\section{DISCUSIÓN}

Los pacientes con signos y síntomas producto de desórdenes temporomandibulares deben ser abordados cuidadosamente, con previa determinación de un diagnóstico específico y plan de tratamiento integral. Las alteraciones oclusales, entre ellas las ocasionadas por hábitos parafuncionales desencadenan desordenes temporomandibulares. La exploración topográfica para la valoración de la sintomatología temporomandibular crea un mapa del dolor que nos permite identificar e interceptar el tipo de problema articular. ${ }^{11}$ La prescripción de analgésicos va a controlar el dolor en los primeros días, luego con la colocación de bite turbos se obtuvo una reposición del cóndilo; que además se apaciguó la sintomatología a lo largo el tratamiento de ortodoncia.

La combinación de las disciplinas como ortodoncia y rehabilitación oral que resuelvan la etiología de estas patologías se encuentra avalada por la literatura, con la ortodoncia se logra una mejor coordinación de las arcadas dentales. Con el tratamiento restaurador una correcta guía incisiva, llaves caninas, over jet, over bite, estabilidad de la oclusión y una estética agradable, (Figura 6) por ende una mejor función, sin trabas en sus movimientos dinámicos que nos ayudará a mantener una estabilidad ortopédica a nivel de la ATM, de esta manera, el cóndilo quedará en una posición favorable dentro de su cavidad glenoidea para que su disco articular lo acompañe en todos sus movimientos de céntrica y excéntrica. ${ }^{9}$

No es viable resolver el caso solo con rehabilitación, porque la mordida borde a borde no proporciona espacio para el material restaurador y al querer resolverlo seríamos muy invasivos con los tejidos sanos del paciente; por otro lado, resolverlo solo con ortodoncia significaría dejar al paciente sin guías para los movimientos de excéntrica. ${ }^{7}$

La férula nocturna utilizada al final del tratamiento 


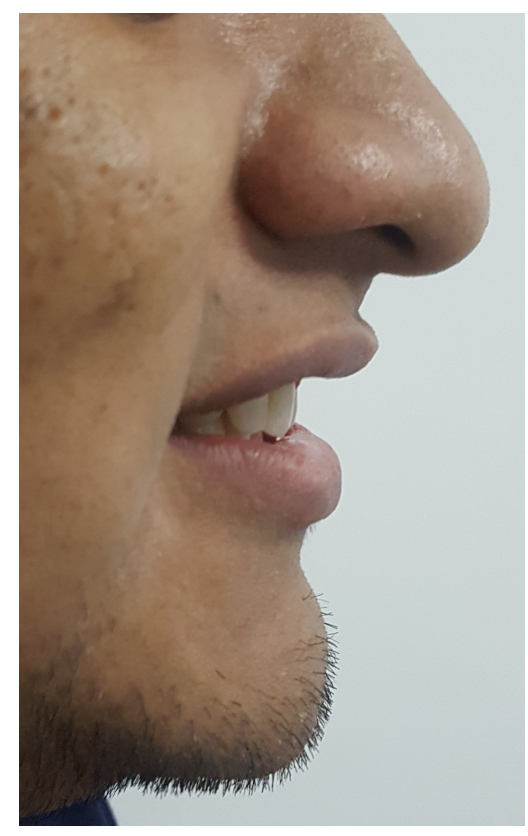

Fig. 4.

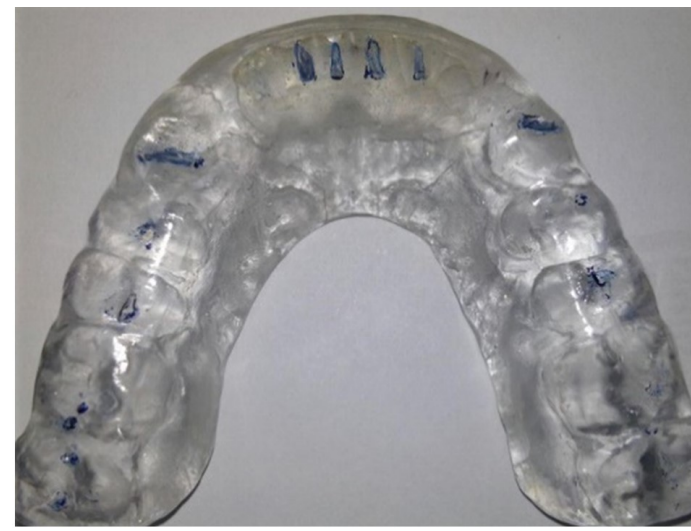

Fig. 5.

disminuye la sintomatología de la articulación temporomandibular, al mantener sus componentes en una posición ortopédica. ${ }^{12}$

Los expertos no se han puesto de acuerdo en la eficacia del tratamiento de ortodoncia en el tratamiento de los trastornos temporomandibulares, existen opiniones a favor y en contra, con el caso descrito presentamos una opción terapéutica combinada, interdisciplinaria que mediante la estabilidad oclusal se alcanza una salud articular.

CONFLICTO DE INTERESES: Los autores no manifiesta ningún conflicto de interés.

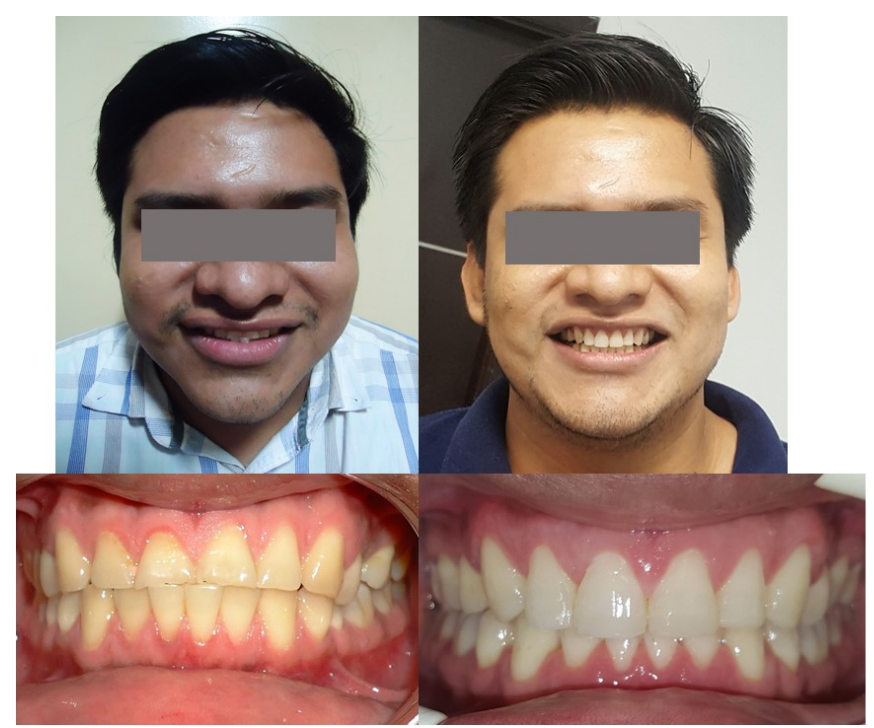

Fig. 6.

\section{Referencias Bibliográficas}

1 Okeson J. Tratamiento de los desordenes temporomandibulares y oclusión. 4th ed. Sao Paulo: Artes médicas; 2000.

2 Sereni A, Borelli P. Programa preventivo para el dolor orofacial y la disfunción de la articulación temporomandibular (ATM). SIMTEC. 2008 Octubre; 2(308).

3 Agnieszka A. Psychosocial Predictors of Bruxism. BioMed Research International. 2019 Octubre.

4 Carlsson G. Some dogmas related to prosthodontics, temporomandibular disorders and occlusion. Acta Odontológica Scandinavica. 2010 Agosto; 68.

5 Tomislav M. Temporomandibular Disorders and Orthodontic Treatment. ResearchGate. 2012 March.

6 Eric R. Diagnostic Criteria for Temporomandibular Disorders (DC/TMD). J Oral Facial Pain Headache. 2.015 June; 28(1).

7 Garrigós P, Elizagaray G, Domínguez A. Temporomandibular disorders: improving outcomes using a multidisciplinary approach. Journal of Multidisciplinary Healthcare. 2019 Septiembre; 12.

8 Simona S. Fixed Orthodontic Therapy in Temporomandibular Disorder (TMD) Treatment. CRANIO. 2014 February; 28(1).

9 Kinzinger A. Effects of orthodontic treatment with fixed functional orthopaedic appliances on the disc-condyle relationship in the temporomandibular joint: a magnetic resonance imaging study. Dentomaxillofacial Radiology. 2006; 35 .

10 Sabine H. Temporomandibular joint remodeling in adolescents and young adults during Herbst treatment. Dentofacial Orthopedics. 1999 June; 115(6).

11 Collante C, Omar Á, Altamirano R. Diagnóstico diferencial 
Tratamiento interdisciplinario de paciente adulto con trastornos de la articulación temporomandibular. Reporte de caso clínico.

de los transtornos témposomandibulares (T.T. M.). Divulgación. 2013 Octubre; 2(6).

12 Valenzuela Y, Moreno T, García F. Evaluación de los Cambios sintomatológicos a través del mapa del dolor después del uso de una férula oclusal para desprogramar la articulación temporomandibular. Rev Med UAS. 2016 Abril-Junio; $7(2)$.

Recibido: 01 de Agosto 2020

Aceptado: 29 de Agosto del 2020 
